\title{
Eisen - ein zweischneidiges Schwert? Systematischer Review zur klinischen Bedeutung eines essenziellen Spurenelements
}

\author{
Reinhard Saller1', Christine Römer-Lüthi'2, Reto Brignoli3, Remy Meier ${ }^{4}$ \\ 1 UniversitätsSpital, Institut für Naturheilkunde, $\mathrm{CH}$-Zürich; ${ }^{2}$ AusbildungszentrumUniversitätsspital, $\mathrm{CH}-\mathrm{Bern}$; ${ }^{3}$ Tradyser $\mathrm{GmbH}$, CH-Rüschlikon; \\ 4Medizinische Universitätsklinik, Abteilung für Gastroenterologie, CH-Liestal
}

\section{isen} isen ist ein Metall mit dem chemischen Symbol Fe (lateinisch Ferrum). Der Name Eisen ist entweder auf das urkeltische Wort „isorai“ oder auf das indogermanische Wort „eison“ (glänzend) zurückzuführen.

Eisen ist für fast alle Lebewesen ein essenzielles Spurenelement. Als Zentralatom im Hämoglobin und Myoglobin transportiert und speichert Eisen den Sauerstoff. In diesen Proteinen ist

\section{Was ist Eisen?}

das Eisenatom von einem Porphyrinring umgeben, dem Häm, das strukturell mit dem „Blattgrün“ der Pflanzen (Chlorophyll) verwandt ist. Weiter ist Eisen Bestandteil von Eisen-SchwefelKomplexen in vielen Enzymen, beispielsweise in Nitrogenasen und Hydrogenasen. Als andere wichtige Klasse der Eisenenzyme sind die sog. Nicht-HämEisenenzyme zu nennen, beispielsweise Methan-Monooxygenase, Ribonukleotid-Reduktase und Hämerythrin. Diese Proteine nehmen in verschiedenen Organismen Aufgaben der Sauerstoffaktivierung, des Sauerstofftransports, der Redoxreaktionen und Hydrolysen wahr. Ebenso wichtig ist dreiwertiges Eisen als Zentralion im Enzym Katalase, das in den Peroxisomen der Zellen das im Stoffwechsel entstehende Zellgift Wasserstoffperoxid abbaut. Bakterien, die in den Organismus eindringen, sind oft darauf angewiesen, das Eisen dieses Organismus zu nutzen. Aus diesem Grund ist das „Verbergen” von Eisen ein Abwehrmechanismus des Körpers.

Bei einem Eisenmangel kann es zu Blutarmut und Abwehrschwäche kom-
Hintergrund: Eisen ist für fast alle Lebewesen ein essenzielles Spurenelement. Als Zentralatom im Hämoglobin und Myoglobin transportiert und speichert Eisen den Sauerstoff. Der Eisenstoffwechsel im Organismus wird normalerweise durch verschiedene Regelkreise strikt kontrolliert, da Eisen einerseits bei vielen Reaktionen eine wichtige Rolle spielt, andererseits aber auch giftig wirken kann. Zielsetzung: Erstellung eines systematischen Review zur klinischen Bedeutung von Eisen. Methoden: Systematische Analyse und Bewertung von Humanstudien (prospektive Doppelblindstudien, epidemiologische und retrospektive Studien, kurzfristige biochemische/hämatologische Studien («Surrogate Markers»)) der letzten 10 Jahre aus den gängigen elektronischen Datenbanken sowie der Angaben von Standardwerken und publizierten Monographien. Ergebnisse und Schlussfolgerungen: Eisen in Fleisch und Meeresfrüchten ist am besten verwertbar. Ebenfalls reich an Eisen sind Gemüse und Früchte - dieses Eisen kann der Körper aber weniger gut aufnehmen und verarbeiten. Vitamin C und Zitronensäure fördern die Aufnahme von Eisen im Darm. Eine Eisenüberladung des Organismus führt mit der Zeit zur Zerstörung der betroffenen Organe (Hämochromatose). Typische Folgen von Eisenspeicherkrankheiten sind Leberzirrhose oder eine Fibrose des Pankreas mit Diabetes mellitus. Eisenmangelanämie ist die häufigste Störung des Eisenstoffwechsels. Die Therapie erfolgt in erster Linie durch die Beseitigung der Ursachen. Ausserdem müssen die Eisenspeicher des Körpers aufgefüllt werden, in der Regel mit einer an Eisen reichen Ernährung und Eisenpräparaten. Die unnötige Einnahme von Eisen kann eventuell Entzündungen oder Infektionen fördern. Eisenpräparate sollten deshalb nur nach einer Abklärung und Verschreibung durch den Arzt eingenommen werden. Bei verschiedenen Erkrankungen wie Typ 2-Diabetes, Alzheimerdemenz oder Morbus Parkinson wird diskutiert, ob ein Eisenüberangebot Bedeutung haben könnte; therapeutische Folgerungen lassen sich daraus (noch) nicht ableiten.

Schlüsselwörter: Eisen, Ernährung, Eisenmangel, Eisenüberladung

\section{Iron - a Two-edged Sword?}

\section{Systematic Review of the Clinical Significance of an Essential Trace Element}

Background: Iron is an essential trace element for almost all forms of life. As the central atom in haemoglobin and myoglobin, iron transports and stores oxygen. Iron metabolism in the organism is normally strictly regulated by a variety of control systems, as iron is not only important in many reactions, but can also act as a poison. Objective: To conduct a systematic review of the clinical significance of long iron. Methods: Systematic analysis and evaluation of human studies - including prospective double blind studies, epidemiological and retrospective studies, short term biochemical and haematological studies with surrogate markers - performed in the last 10 years and found in major electronic data bases, coupled to information in standard works and published monographs. Results and Conclusion: Iron in meat or sea food is the most readily assimilated. Although vegetables and fruit are also rich in iron, this is in a form which is less easily taken up and processed. Vitamin $C$ and citric acid enhance the uptake of iron in the intestine. However, if an organism is overloaded with iron, this can destroy the affected organs with time ("haemochromatosis"). Iron storage diseases typically lead to liver cirrhosis or pancreatic fibrosis with diabetes mellitus. Iron deficiency anaemia is the most frequent abnormality in iron metabolism. This is primarily treated by removing the causes. In addition, the iron stores in the body must be filled up, usually with iron rich food and iron preparations. Unnecessary iron intake may exacerbate inflammation or infection so that iron preparations should only be taken after discussion with a doctor and on prescription. It is currently under discussion whether excessive iron supply may be an important factor in a variety of diseases, including type 2 diabetes, Alzheimer's disease and Parkinson's disease. This has not (yet) led to any changes in therapy.

Key words: Iron, nutrition, iron deficiency, iron overload 


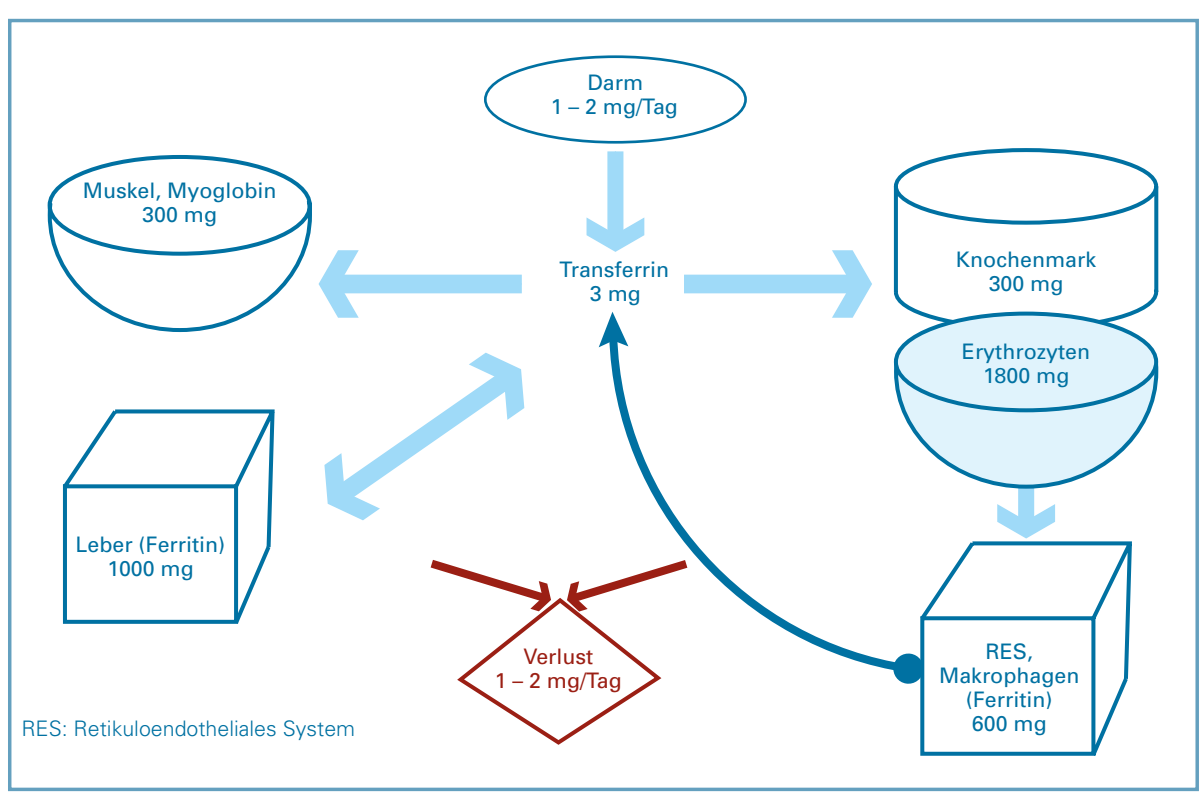

Abb. 1. Resorption und Verteilung von Eisen im Körper.

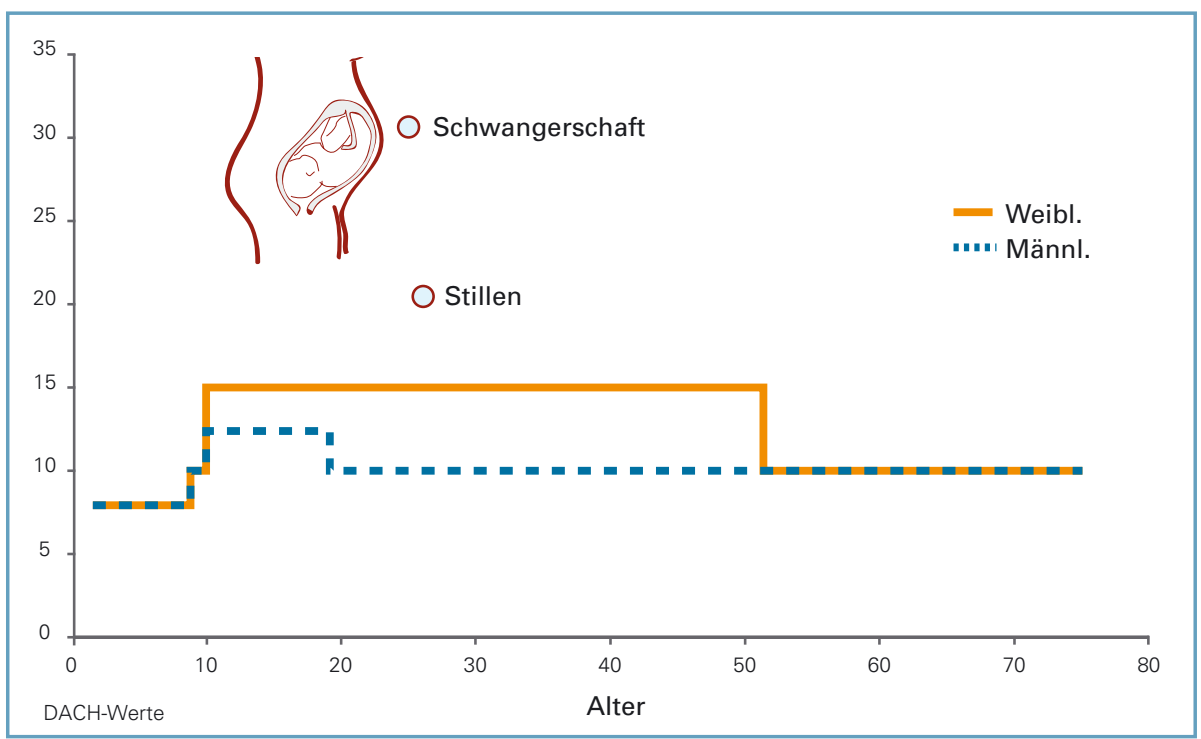

Abb. 2. Empfohlene Eisenzufuhr in mg pro Tag, nach Alter und Geschlecht [1].

men; bei kleinen Kindern treten Entwicklungsstörungen auf.

Obwohl Eisen ein wichtiges Spurenelement ist, kann zu viel Eisen im Körper giftig sein. Zu grosse Mengen an freien Eisenionen reagieren mit Peroxiden, wobei freie Radikale entstehen. Im Normalzustand werden die freien Radikale durch körpereigene Prozesse kontrolliert und abgebaut. Bei einer Eisenüberladung kann es aber je nach betroffenem Organ zu einer Leberzirrhose, zu einer Pankreaszerstörung (Bronzediabetes) und anderen Organstörungen kommen.
Auf Eisenverwertungsstörungen, die durch fehlerhafte Hämoglobinmoleküle entstehen (Sichelzellanämie, Thalassämien), und Eisenüberlastungen durch Hämolyse (zum Beispiel bei Malaria) wird hier nicht näher eingegangen.

\section{Der normale Eisenstoffwechsel}

Eisen ist von zentraler Bedeutung beim Elektronentransport und bei verschiedenen Oxidations-ReduktionsReaktionen. Freies Eisen ist sehr reak- tiv und deshalb toxisch für die Zellen. Weil Eisen in verschiedenen Reaktionen eine so wichtige Rolle ausübt, gleichzeitig aber giftig ist, untersteht der Eisenstoffwechsel einer strikten Kontrolle. Die Zellen verfügen einerseits über einen ausgeprägten Oxidationsschutz, andererseits wird das Eisen ausschliesslich an Proteine gebunden, transportiert und gespeichert.

\section{Eisenbedarf}

$70 \%$ des Eisens im Organismus sind im Hämoglobin enthalten $(500 \mathrm{ml}$ Blut enthalten 250 mg Eisen). Durch kleine Blutungen und Abschilferung von Schleimhautzellen verlieren Erwachsene ca. $1 \mathrm{mg}$ Eisen pro Tag. Bei Frauen kommt es durch die Menstruation zu einem zusätzlichen monatlichen Eisenverlust von 3 bis $60 \mathrm{mg}$. Bei jeder Schwangerschaft gibt die Frau $500 \mathrm{mg}$ Eisen an das wachsende Kind ab (Abbildung 1).

Mit einer üblichen Ernährung nimmt man ca. $20 \mathrm{mg}$ Eisen pro Tag auf. Die Aufnahme des Eisens im Dünndarm wird reguliert, um den individuellen Eisenverlust $\mathrm{zu}$ kompensieren. Ein Mann absorbiert ca. 5\% des Eisens, das er mit der Nahrung zu sich nimmt. In der Schwangerschaft und Stillzeit steigt der Eisenbedarf stark an (Abbildung 2).

\section{Resorption und Transport von Eisen}

Häm in Fleisch und Meeresfrüchten ist die am besten bioverfügbare Form von Eisen; es wird im oberen Dünndarm resorbiert. Anorganisches Eisen kommt in der Nahrung vor allem als dreiwertiges Eisen in Gemüsen und Früchten vor (Tabelle 1).

Wie viel Eisen aus der Nahrung resorbiert wird, hängt von der Wertigkeit des Eisens und der Anwesenheit anderer Nahrungsbestandteile ab. Substanzen wie Tannine, Oxalsäure und Phosphate hemmen die Eisenresorption, also zum Beispiel Nahrungsmittel wie Weizenkleie, Schwarztee und Sojaprodukte. Auch Arzneimittel wie Aspirin und Antazida hemmen die Aufnahme von Eisen. Resorptionsfördernd wirken Vitamin C und Zitronensäure.

Damit Eisen resorbiert werden kann, muss es durch Magensäure aus 
Tab. 1. Eisengehalt in verschiedenen Nahrungsmitteln (angepasst von www.mayohealth.org/mayo/pted/htm/iron.htm)

\begin{tabular}{|lc|}
\hline Sehr gute Quellen & mg Eisen pro Portion $(\mathbf{8 0} \mathbf{g})$ \\
\hline Muscheln & $12-24$ \\
\hline Austern & $6-12$ \\
\hline Leber und Innereien & $5-26$ \\
\hline Rind-, Schweine- oder Schaffleisch & $2-23$ \\
\hline Geflügel & $1-22$ \\
\hline Fisch & $0,5-21$ \\
\hline Gute Quellen & mg Eisen pro Portion \\
\hline Vollkornbrot & $1 \mathrm{mg}$ pro Scheibe \\
\hline Pasta & $2 \mathrm{mg}$ pro Tasse \\
\hline Grüne Gemüse: Spinat, Artischocken, Kohlarten & $1-3 \mathrm{mg}$ pro halbe Tasse \\
\hline Dörrfrüchte: Rosinen, Feigen, Aprikosen, Birnen etc. & $1-3 \mathrm{mg}$ pro halbe Tasse \\
\hline Nüsse: Mandeln, Cashews, Erdnüsse etc. & $1 \mathrm{mg}$ pro $30 \mathrm{~g}$ \\
\hline
\end{tabular}

den Bindungen freigesetzt werden und durch reduzierende Verbindungen, wie z.B. Vitamin C oder membrangebundene Enzyme, zum zweiwertigen Eisen reduziert werden. Mithilfe eines speziellen Transportproteins für Metalle gelangt Eisen in die Schleimhautzellen des Darms und immer gebunden an Proteine über spezifische Transportwege von dort ins Blut.

Im Blut wird Eisen - durch Hephästin und Coeruloplasmin wieder zu dreiwertigem Eisen oxidiert - an Transferrin gebunden. In dieser Form gelangt es zu den Orten des Bedarfs und über den Transferrinrezeptor in die Gewebezelle. Bei gesteigertem Eisenbedarf wird die Transferrinsynthese stimuliert und Eisen dadurch effizienter aus dem Dünndarm resorbiert. Diese Steuerung erfolgt über verschiedene Faktoren, unter anderem Enzyme sowie die mRNA von Transferrin und Ferritin. In den Zellen wird Eisen als Ferritin eingelagert; ein Molekül Ferritin kann ca. 4500 Eisenmoleküle speichern (Abbildung 3).

Der Eisenspiegel wird vor allem durch das erst kürzlich entdeckte Peptid Hepcidin [2] reguliert; dieses Peptid hat auch antimikrobielle Eigenschaften: Es wirkt einerseits direkt antibakteriell, andererseits dadurch, dass es den Bakterien das dringend benötigte Eisen entzieht [3]. Hepcidin wird in der Leber synthetisiert und wahrscheinlich in der Niere abgebaut bzw. im Harn ausgeschieden [4, 5]. Ist der Eisenspiegel zu niedrig, dann bremst die Leber ihre Hepcidinproduktion; als Folge wird im Darm mehr Eisen resorbiert. Ist der Eisenspiegel zu hoch, dann steigert die Leber ihre Hepcidinproduktion und die Eisenresorption im Darm nimmt ab. Man hat Hepcidin deswegen auch als Hormon des Eisenstoffwechsels bezeich-
Abb. 3. Enteraler und systemischer Eisentransport. net. Auch die Freisetzung von Eisen aus den Zellen des retikuloendothelialen Systems (RES) wird durch Hepcidin gesteuert [6].

\section{Diagnose des Eisenstatus}

Will man sich einen Überblick über die Versorgung des Organismus mit Eisen verschaffen, müssen Serum-Eisen, Serum-Transferrin und Serum-Ferritin bestimmt werden. Die Ferritinwerte korrelieren mit den Eisenreserven im Gewebe: $1 \mathrm{mg} / \mathrm{l}$ Ferritin entspricht $10 \mathrm{mg}$ Eisen im Gewebe. Bei akuten Entzündungen stimmt diese Korrelation allerdings nicht, weil Ferritin ein Akute-Phase-Protein ist und daher bei akuten Entzündungen die Ferritinwerte erhöht sind. Die Leber ist ein wichtiger Eisenspeicher und enthält viel Ferritin, deshalb steigen die Ferritinwerte bei Leberzellnekrosen ebenfalls an.

Bei chronisch entzündlichen Prozessen, wie zum Beispiel rheumatoider Arthritis oder Krebserkrankungen, kommt es oft zu Eisenverteilungsstörungen. Sie beruhen darauf, dass zu wenig Transferrin gebildet und/oder

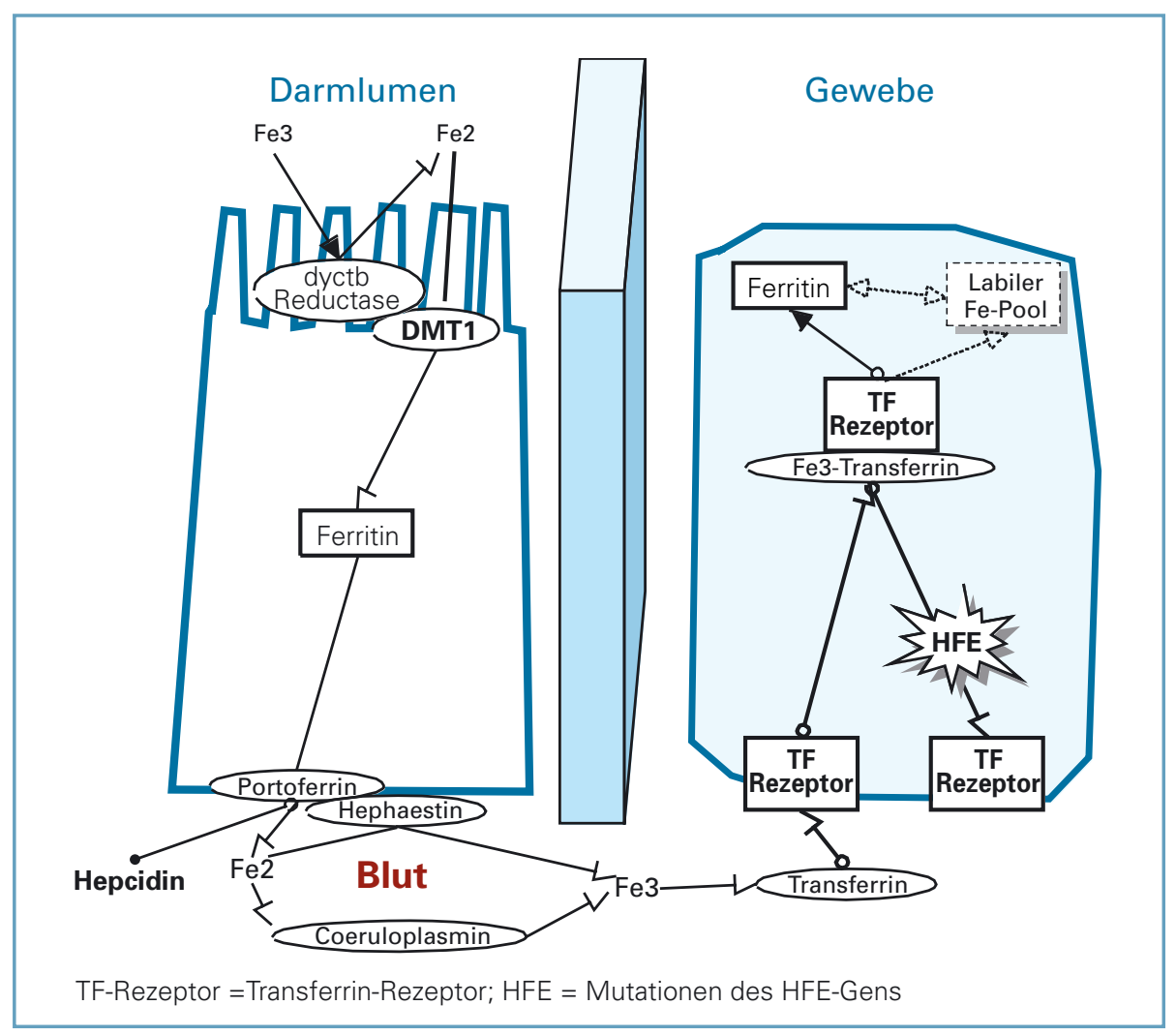


das Transferrin zu wenig beladen wird. Dies führt zu Entzündungs- bzw. Infekt- oder Tumoranämien, die mit einer normalen bis erhöhten Ferritinkonzentration und erniedrigten Transferrinwerten einhergehen. Eisenverteilungsstörungen können nur teilweise mit der Transferrinsättigung erkannt werden. Die Transferrinsättigung wird zusätzlich durch den stark schwankenden Rhythmus der Serum-EisenKonzentration im Verlauf des Tages beeinflusst.

Die Eisenkonzentration in der Leber ist altersabhängig und wird als hepatic iron index (HII) ausgedrückt. Berechnet wird der HII als Eisenkonzentration in $\mathrm{mmol} / \mathrm{g}$ geteilt durch das Alter in Jahren. Der HII ist normalerweise geringer als 1,5 .

Die Serumkonzentration von löslichen Transferrinrezeptoren (sTfR) spiegelt die Rezeptorkonzentration auf den Zellmembranen wider. 80-95\% der Transferrinrezeptoren (TfR) befinden sich auf Zellen des erythropoietischen Systems. Daher zeigt die sTfRKonzentration den Eisenbedarf, aber auch die Anzahl dieser Zellen an. Bei Eisenmangel steigt die sTfR-Konzentration, da die Erythropoesezellen mehr TfR exprimieren. Bei hämolytischen Anämien nimmt die sTfR-Konzentration ebenfalls zu, weil sich die Anzahl der Erythropoesezellen und damit der TfR erhöht. Im Gegensatz zum Serum-Ferritin wird die Konzentration von sTfR nicht durch akute Leberfunktionsstörungen oder maligne Tumoren beeinflusst. Das ermöglicht die diagnostische Beurteilung, ob eine Anämie von einer chronischen Erkrankung ausgelöst wird oder durch einen Eisenmangel. Der sTfR-Wert wird in der Regel nicht isoliert, sondern gemeinsam mit Serum-Eisen, SerumTransferrin, Serum-Ferritin und weiteren Laborparametern beurteilt. Mit dem sTfR-Wert kann man den aktuellen Eisenbedarf abschätzen, während Ferritin die vorhandenen Eisenspeicher widerspiegelt. Beide Werte zusammen liefern ein genaueres Bild des Eisenstatus, den man mittels des sTfRIndex auch quantifizieren kann (Referenzbereich: 0,9-2,8 mg/l) (Tabelle 2).

Von den Störungen des Eisenstoffwechsels bei der Resorption, beim

Tab. 2. Eisenstoffwechsel und löslicher Transferrinrezeptor (sTfR)

\begin{tabular}{|llll}
\hline Pathologie & Ferritin & sTfR & Retikulozyten \\
\hline Eisenmangel & erniedrigt & erhöht & erniedrigt - normal \\
\hline Infekt- / Tumoranämie & normal - erhöht & normal & normal \\
\hline Erythrozytenreifungsstörung & normal - erhöht & erhöht & normal \\
\hline Hämolytische Anämie & normal - erhöht & erhöht & erhöht
\end{tabular}

Transport oder bei der Speicherung lassen sich Eisenverwertungsstörungen abgrenzen, die durch Prozesse in den Zellen verursacht werden. In den Retikulozyten des Knochenmarks baut das Enzym Ferrochelatase das aufgenommene Eisen in Protoporphyrin IX ein und es entsteht das Häm. Bei einem Eisenmangel wird alternativ Zink eingebaut: Im Blut kann dann Zink-Protoporphyrin nachgewiesen werden. Am erhöhten Spiegel des Zink-Protoporphyrins bei gleichzeitig erhöhter Ferritinkonzentration lassen sich die Eisenverwertungsstörungen erkennen. Sie kommen zum Beispiel bei chronisch entzündlichen Erkrankungen, Myelodysplasie oder Bleivergiftung vor. Blei hemmt die Ferrochelatase; bei einer Bleivergiftung wird Zink spontan ins Protoporphyrin IX eingebaut.

\section{Eisenspeicherkrankheiten}

Wenn Eisen vermehrt im Gewebe abgelagert wird, zeigt sich dies mikroskopisch als Hämosiderose. Der Eisenüberschuss schädigt das Gewebe, und im Endzustand fibrosiert das Gewebe. Eine Hämosiderose in Kombination mit Gewebeschäden wird als Hämochromatose bezeichnet. Hämochromatosen können primär als erbliche Erkrankung oder sekundär auftreten, zum Beispiel als Folge von vermehrten Bluttransfusionen oder überhöhter Eiseneinnahme.

Bei verschiedenen Volksgruppen im südlichen Afrika sind histologische Hinweise für eine Eisenüberladung im Körper besonders häufig beobachtet worden, weshalb diese Erkrankung auch als „Bantu-Siderose“ in die Literatur eingegangen ist. Ursache dafür ist der häufige Genuss von Bier, das traditionell in Eisenfässern gebraut wird und deshalb einen hohen Eisengehalt aufweist.

Die hereditäre Hämochromatose ist die häufigste autosomal rezessiv vererbte Erbkrankheit. Bei etwa 10\% der Bevölkerung liegt eine entsprechende Mutation des HFE-Gens vor (C282Y, H63D9). Allerdings treten nur Symptome auf, wenn der Gendefekt homozygot vorliegt, und dies auch nur bei etwa $25 \%$ der homozygoten Hämochromatose-Genträger.

Der Gendefekt führt dazu, dass Eisen im Dünndarm ungehemmt resorbiert wird. Als Folge werden verschiedene Organe mit Eisen überladen und es entstehen entsprechende Gewebeschäden:

- Leber: Entzündung, Fibrosierung bis zur Zirrhose und hepatozelluläres Karzinom

- Pankreas: Fibrosierung, Diabetes mellitus („Bronzediabetes“ wegen dunkel verfärbter Haut)

- Haut: Hyperpigmentierung

- Herz: Kardiomyopathie, Herzinsuffizienz, Herzrhythmusstörungen

- Gelenke: Arthropathie (Pyrophosphat-Arthritis)

- Endokrine Organe: Schädigung der Hypophyse und der Geschlechtsorgane

Nur noch selten präsentieren sich Patienten mit hereditärer Hämochromatose mit den klassischen drei Symptomen Leberzirrhose, hyperpigmentierte Haut und Diabetes mellitus („Bronzediabetes“). Es gibt aber auch heute noch immer wieder Patienten, die jahre- bis jahrzehntelang an Müdigkeit, Unwohlsein oder Gelenkschmerzen leiden, bevor die Diagnose der hereditären Hämochromatose gestellt wird. Die Symptome sind leider unspezifisch und viel häufiger auf andere Krankheiten zurückzuführen. Ausschlaggebend für eine vertiefte Abklärung sind oft 
erhöhte Transaminasen oder eine vergrösserte Leber [8].

Heute sollte die hereditäre Hämochromatose früh erkannt werden, denn Organschäden treten erst nach Jahren der Eisenüberladung auf. Wird die Diagnose frühzeitig gestellt und werden die Patienten mit regelmässigen Aderlässen behandelt, so haben sie eine normale Lebenserwartung. Ein Screening mittels Bestimmung der Transferrinsättigung ist kosteneffektiv. Beträgt diese über 60 Prozent, so wird das Ferritin bestimmt. Zur endgültigen Diagnose ist meist eine Leberbiopsie notwendig. Wird bei einem Patienten die hereditäre Hämochromatose diagnostiziert, sollten auch seine Familienangehörigen beraten und gescreent werden.

Das neu entdeckte „Eisenhormon“ Hepcidin, das in der Leber produziert wird und im Dünndarm die Eisenaufnahme hemmt, scheint eine zentrale Rolle im Eisenstoffwechsel zu spielen. Mutationen im Hepcidin-Gen verursachen die juvenile hereditäre Hämochromatose, bei der die Symptome schon im Jugendalter auftreten [9].

\section{Eisenmangelanämie}

Die Häufigkeit von Eisenmangelanämien ist sehr unterschiedlich, sie wird in den USA auf 4,1\% der Erwachsenen geschätzt; betroffen sind ca. $20 \%$ der Frauen mit Menstruation und ca. 2\% der Männer [10]. In Frankreich hatten $23 \%$ der menstruierenden Frauen (28\% der Frauen mit IUD!) und 5,3\% der postmenopausalen eine Erschöpfung der Eisenredepots (Ferritin <15 mg/l); eine Anämie konnte bei 4,4\% der menstruierenden Frauen und 1\% der postmenopausalen Frauen festgestellt werden. Bei mehr als der Hälfte der Frauen liegt die tägliche Eisenzufuhr deutlich unter den empfohlenen Tagesmengen [11]. In Deutschland findet man bei etwa 0,6\% der Erwachsenen Hinweise auf eine Eisenmangelanämie, bei Frauen doppelt so häufig wie bei Männern. Die Framinghamstudie zeigte bei den 67- bis 96-jährigen Probanden bei $2,7 \%$ einen Eisenmangel, bei $1,2 \%$ eine Eisenmangelanämie und bei 3\% eine Erschöpfung der Eisendepots (Ferritin <12 $\mu \mathrm{g} /$ l) [12]. Die In- zidenz von Eisenmangel, Eisenmangelanämie und Erschöpfung der Eisendepots war von der Existenz einer chronischen Erkrankung unabhängig.

Beginnender Eisenmangel bleibt oft unbemerkt und kann nur durch LaborBlutkontrollen festgestellt werden. Die Stadien des Eisenmangels werden als prälatenter, latenter oder manifester Eisenmangel bezeichnet, je nachdem, wie viel Eisen im Blut ermittelt wird.

Generell treten beim Eisenmangel eher unspezifische Symptome wie Kopfschmerzen, Konzentrationsstörungen, Abgeschlagenheit und Müdigkeit auf. Schwäche und belastungsabhängige Atemnot können ein Hinweis auf den mangelnden Sauerstoff im Körper sein. Anzeichen für eine Anämie sind neben einer blassen Hautfarbe vor allem blasse Schleimhäute. Ein leichter Eisenmangel kann sich eventuell negativ auf die kognitiven Funktionen von Kindern auswirken; die entsprechenden Studien sind allerdings nicht schlüssig [13, 14].

Weitere Symptome von Eisenmangel sind brüchige Nägel und Haare, diffuser Haarausfall, Rillen in den Fingernägeln, trockene Haut, Juckreiz und immer wieder einreissende Rhagaden der Mundwinkel. Beim Pica-Syndrom entwickeln die Patienten Appetit auf normalerweise Ungeniessbares, wie z.B. Erde. Eine Extremform von Eisenmangel ist das Plummer-Vinson-Syndrom, hier treten infolge einer Schleimhautatrophie im Bereich von Mund, Rachen und Speiseröhre Schluckbeschwerden auf. Ursache ist eine Verminderung eisenhaltiger Enzyme.

Verschiedene klinische Studien zeigen, dass die Komplikationsrate bei akuter koronarer Herzkrankheit bei Patienten mit Anämie massiv höher ist als bei Patienten ohne Anämie. Bei einer Analyse von rund 39'000 Patienten aus verschiedenen Studien fand man heraus, dass die Sterblichkeitsrate bei Hämoglobinwerten unter $14 \mathrm{~g} / \mathrm{dl}$ eindeutig erhöht war; allerdings stieg die Sterblichkeitsrate bei Hämoglobinwerten über $17 \mathrm{~g} / \mathrm{dl}$ ebenfalls an [15]. Ähnliche Zahlen wurden bei Patienten mit akutem Herzinfarkt nach angioplastischen Eingriffen beschrieben: Die Sterblichkeitsrate während des Spitalaufenthalts betrug bei anämischen Patienten $4,6 \%$, bei nichtanämischen Patienten 1,1\% [16].

\section{Ursachen und Risikofaktoren von Eisenmangel}

Besondere Risikogruppen für Eisenmangel sind schwangere Frauen, Heranwachsende und Vegetarier, insbesondere Veganer. Die bestehenden Daten lassen keine allgemeine Empfehlung $\mathrm{zu}$, ob eine Eisensupplementierung bei schwangeren Frauen in unseren Breitengraden sinnvoll ist, obwohl eine neuere Studie diesbezüglich positive Resultate zeigte [17, 18]

Die häufigste Ursache der Eisenmangelanämie sind Eisenverluste durch chronische Blutungen, zum Beispiel infolge der Menstruation bei Frauen. Gehäuft auftretendes Nasenbluten und (okkulte) Blutungen aus dem Verdauungstrakt, zum Beispiel wegen eines Magengeschwürs oder Hämorrhoiden, können ebenfalls zu einer Anämie führen. Eisenmangel kann auch durch eine verminderte Zufuhr von Eisen entstehen, etwa bei Frühgeborenen oder Säuglingen, die lange ausschliesslich mit Milch ernährt werden. Weitere Ursachen sind akute Blutverluste durch Blutspenden, eine Operation oder einen Unfall.

Bei Spitzensportlern im Ausdauerbereich kann es infolge von Rhabdomyolyse durch die Extrembelastung oder wegen gastrointestinaler Mikroblutungen ebenfalls zur Eisenmangelanämie kommen [19, 20, 21]. Bei Sportler(innen) kann deshalb eine niedrig dosierte Eisensupplementierung ins Auge gefasst werden [22].

Verschiedene Magen-Darm-Erkrankungen, wie beispielsweise chronisch entzündliche Darmkrankheiten, Status nach Gastrektomie oder Gastritis infolge einer Helicobacter pylori-Infektion, führen $\mathrm{zu}$ einer verminderten Eisenresorption und damit oft $\mathrm{zu}$ einer Eisenmangelanämie (Abbildung 4). Die betroffenen Patienten bedürfen einer Eisensupplementierung [23, 24]. Auch Hämodialysepatienten benötigen häufig eine intravenöse Eisensupplementierung, damit die Blutverluste, die durch die Dialyse entstehen, kompensiert werden können [25]. 
Therapie der Eisenmangelanämie

Bei der Behandlung der Eisenmangelanämie sollte in erster Linie die Ursache beseitigt werden, zum Beispiel eine Blutungsquelle. Ausserdem müssen die entleerten Eisenspeicher aufgefüllt werden. Dies geschieht zum einen durch eine an Eisen reiche Ernährung, andererseits durch die Einnahme von Eisenpräparaten. Die orale Therapie sollte mindestens drei bis sechs Monate durchgeführt werden, da der Körper Eisen nur in begrenzten Mengen aus dem Darm aufnehmen kann. Die Einnahme erfolgt meist zweimal täglich mit 200-300 mg zweiwertigem Eisen (Eisensulfat) in Tablettenform, oft in Kombination mit Folsäure, um die Aufnahme von Eisen zu verbessern [26]. Frauen mit chronischer Müdigkeit und Ferritinspiegeln unter $50 \mu \mathrm{g} / \mathrm{l}$ können eventuell von einer Eisensupplementierung profitieren [27]. In einer Studie wurde allerdings kein Zusammenhang zwischen tiefen Ferritinspiegeln bei Frauen $(<15 \mu \mathrm{g} / \mathrm{l})$ und der Lebensqualität festgestellt [28].

Eisenpräparate verursachen recht häufig Unverträglichkeitsreaktionen wie Sodbrennen, Brechreiz, Bauchkrämpfe, Diarrhö oder Verstopfung, schwarze Verfärbung der Zunge und der Zähne etc. Um diese Nebenwirkungen zu vermeiden, können gastroresistente Kapseln eingenommen werden. Präparate mit dreiwertigem Eisen sind im Allgemeinen besser verträglich [29].

Sollte eine orale Substitution erwogen werden, ist daran zu denken, dass Eisen und Zink um die Aufnahme in den Körper konkurrieren können [30]; Eisensupplemente hemmen zudem die Resorption von Captopril [31]. Da Schwarztee die Aufnahme von Eisen behindert, sollte Tee frühestens eine Stunde nach einer Mahlzeit oder nach der Einnahme eines Eisenpräparats getrunken werden [32]. Ausserdem sind die Vitamine $\mathrm{B}_{2}, \mathrm{~B}_{6}$ und $\mathrm{B}_{12}$ sowie Folsäure ebenfalls an der Blutbildung beteiligt und sollten, wie auch das Vitamin C, bei einer Substitution nicht vergessen werden.

Bei bedeutender Eisenmangelanämie, zum Beispiel bei Patienten mit chronischem Nierenversagen oder Hämodialysepatienten, können intravenös

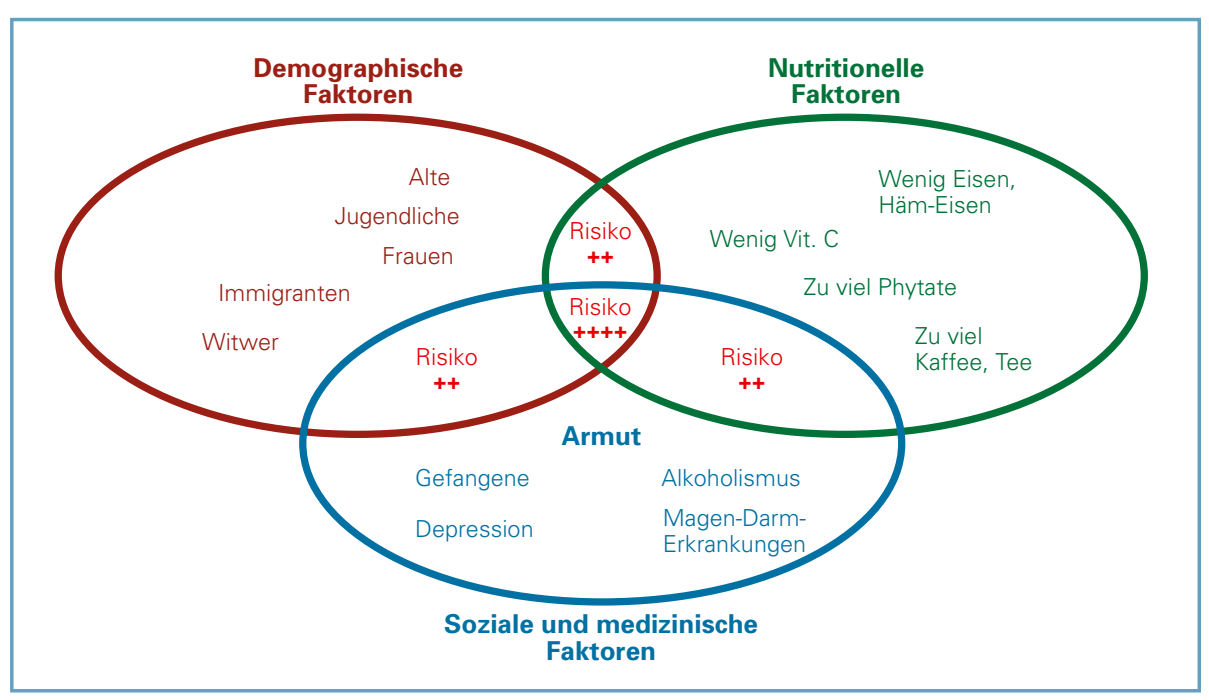

Abb. 4. Risikofaktoren einer Eisenmangelanämie.

applizierte Eisenpräparate eine rasche Eisensättigung erzielen. Zwar ist das Risiko für eine anaphylaktische Reaktion im Rahmen einer solchen Infusion nur klein, trotzdem sollte die intravenöse Substitution von Eisen nur in Einrichtungen vorgenommen werden, wo die Möglichkeit zur kardiopulmonalen Reanimation gegeben ist. Die häufigsten beobachteten Nebenwirkungen sind vorübergehende Störungen der Geschmacksempfindung, Hypotonie, Fieber und Schüttelfrost.

\section{Anämie bei chronischen Erkrankungen}

Die Anämie bei chronischen Erkrankungen ist nach der Eisenmangelanämie die zweithäufigste Anämieform. Ursachen sind meistens Nierenleiden, Krebs, Autoimmunkrankheiten wie rheumatoide Arthritis, (chronische) Infektionen und Abstossreaktionen. Die Pathophysiologie der Anämie bei chronischen Erkrankungen ist komplex (Abbildung 5).

Durch die Wirkung der verschiedenen Substanzen, die bei diesen Erkrankungen vermehrt produziert werden, wird Eisen ins Innere der Zellen verschoben, wo es für die Hämsynthese nicht verfügbar ist. Die Erythroblasten vermehren sich nur noch ungenügend und sie sprechen auch auf die Stimulation durch Erythropoietin nur vermindert an. Die Anämie bei chronischen Erkrankungen ist meistens auch mit einer schlechteren Prognose der Grundkrankheit verknüpft. Eine Korrektur der Anämie verbessert in der Regel die Prognose dieser Grundkrankheit und vice versa.

Die Behandlung beruht auf Transfusionen, Gabe von Erythropoietin und bei eindeutigem Eisenmangel die Gabe von Eisensupplementen. Letztere sind aber mit grosser Vorsicht einzusetzen, da sie die Infektanfälligkeit erhöhen können.

\section{Andere Störungen des Eisenhaushalts}

Die Acoeruloplasminämie ist eine seltene Erbkrankheit mit defektem Coeruloplasmin, einem Glykoprotein mit Bindungs- und Transportfunktion für Kupfer. Coeruloplasmin reduziert dreiwertiges Eisen des intrazellulären Ferritinpools zu zweiwertigem Eisen, um es aus der Zelle zu schaffen und dem Transferrin zu übergeben. Durch den Mangel an Coeruloplasmin entwickelt sich einerseits eine (scheinbare) Eisenmangelanämie, andererseits leiden die Patienten an extrapyramidalen Symptomen, zerebellarer Ataxie, Demenz und Diabetes mellitus.

Bei der Krankheit „Hepatische Eisenüberladung bei Insulinresistenz” (Insulin resistance-associated hepatic iron overload, IR-HIO) kombinieren sich Störungen des Eisenstoffwechsels 


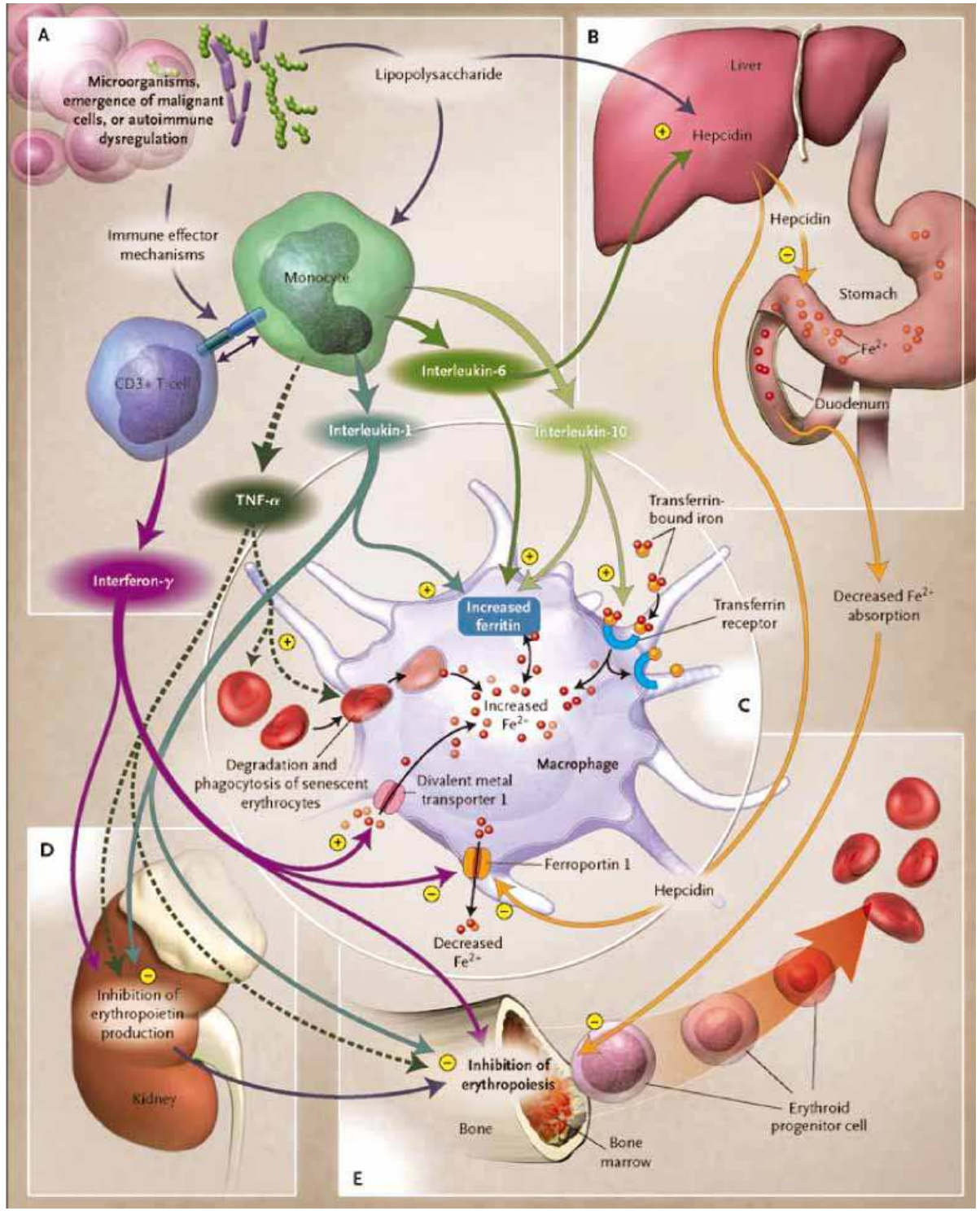

Abb. 5. Pathophysiologie der Anämie bei chronischen Erkrankungen.

(Hyperferritinämie bei normaler Transferrinsättigung), eine Verfettung der Leber und das metabolische Syndrom (Adipositas, Hyperlipidämie, abnorme Glukosetoleranz und Hypertonie). Die Eisenkumulation findet primär in den Hepatozyten, insbesondere in den Sinusoidalzellen statt (ist bei Hämochromatose eher selten). Ca. $60 \%$ der Fälle entwickeln eine Leberfibrose.

Beim Typ 2-Diabetes, speziell bei Schwarzen, wird diskutiert, inwiefern erhöhte Eisendepots an der Entstehung der Krankheit beteiligt sein könnten [33]; experimentell bewirkte die Behandlung mit dem Eisenchelator Deferoxamin oder Phlebotomie eine Besserung der vaskulären Folgeerscheinungen. Therapeutische Studien ablagerung in den nigrostriatalen Strukturen beschrieben, was zu einer oxidativen Beschleunigung der Krankheit führen könnte [39, 40].

Ein Zusammenhang zwischen Arteriosklerose und gesteigerter Eisenbelastung wird kontrovers diskutiert [41, 42].

\section{Schlussfolgerungen}

Der Eisenstoffwechsel wird im normalen Organismus durch verschiedene Regelkreise strikt kontrolliert, da Eisen einerseits bei vielen Reaktionen eine zentrale Rolle spielt, andererseits aber auch potenziell toxisch ist. Die vermehrte Speicherung von Eisen tritt bei Erbkrankheiten wie der Hämochromatose auf.

In Fleisch und Meeresfrüchten ist am meisten Eisen in einer gut bioverfügbaren Form vorhanden; dreiwertiges Eisen kommt vorwiegend in Gemüse und Früchten vor.

Eisenverluste durch chronische Blutungen sind die häufigste Ursache von Eisenmangelanämien. Therapeutisch sollten in erster Linie die Ursachen beseitigt werden. Ausserdem müssen mit entsprechender Ernährung und oraler Eisensupplementierung die Eisenspeicher aufgefüllt werden. Bei einer hochgradigen Eisenmangelanämie lassen sich die Eisenspeicher durch die intravenöse Gabe von Eisensaccharose oder Eisengluconat rasch auffüllen.

Die unnötige Einnahme von Eisen kann eventuell entzündliche oder infektiöse Prozesse begünstigen. Eisenpräparate sollten deshalb erst nach einer Abklärung und Verschreibung durch den Arzt eingenommen werden.

Anämien bei chronischen Erkrankungen entstehen unter anderem wegen einer Verschiebung des Eisens in Zellen, wo es für die Hämsynthese nicht verfügbar ist. Die Behandlung dieser Anämien ist komplex und muss spezifisch auf den einzelnen Patienten ausgerichtet werden.

Bei verschiedenen Erkrankungen wie Typ 2-Diabetes, Alzheimerdemenz, Morbus Parkinson oder Arteriosklerose wird diskutiert, welche Bedeutung ein (lokales) Eisenüberangebot 
haben könnte; therapeutische Folgerungen lassen sich gegenwärtig jedoch daraus nicht ableiten, auch mangels entsprechender Studien.

This paper is an updated summary of an expert-workshop sponsored by BIOMED AG, $\mathrm{CH}$-Dübendorf.

\section{Literatur}

1 DACH Referenzwerte für die Nährstoffzufuhr. 1. Auflage 2000, Herausgeber: Deutsche Gesellschaft für Ernährung DGE, Österreichische Gesellschaft für Ernährung ÖGE, Schweizerische Gesellschaft für Ernährungsforschung SGE, Schweizerische Vereinigung für Ernährung SVE. Seite 230-231

2 Robson KJ: Hepcidin and its role in iron absorption. Gut 2004; 53; 617-619

3 Editorial. Hepcidin: inflammation's iron curtain Rheumatologv 2004: 43: 1323-325

4 Kulaksiz H, Gehrke SG, Janetzko A, Rost D, Bruckner T, Kallinowski B, Stremmel W. Prohepcidin: expression and cell specific localisation in the liver and its regulation in hereditary haemochromatosis, chronic renal insufficiency, and renal anaemia. Gut (2004 May) 53(5): 735-43

5 Detivaud L, Nemeth E, Boudjema K, Turlin B, Troadec MB, Leroyer P, Ropert M, Jacquelinet S, Courselaud B, Ganz T, Brissot P, Loreal O: Hepcidin levels in humans are correlated with hepatic iron stores, hemoglobin levels, and hepatic function. Blood (2005 Jul 15) 106(2): 746-8

6 Rouault TA: How Mammals Acquire and Distribute Iron Needed for Oxygen-Based Metabolism. PLoS Biology. http://biology.plosjournals.org: 2003 Volume, Issue 3, Page 326-328

7 http://www-klinik.uni-mainz.de/Zentrallabor/ Lab-Web/PCR_Analytik.htm; zuletzt aktualisiert am 02.06.2005

8 Heim M: Hereditäre Hämochromatose: Fortschritte - Auswirkungen. Hémochromatose héréditaire: progrès - résultats. Schweiz Med Forum 2004; 4:971-975

9 Roetto A, Daraio F, Porporato P, Caruso R, Cox TM, Cazzola M, Gasparini P, Piperno A, Camaschella C: Screening hepcidin for mutations in juvenile hemochromatosis: identification of a new mutation (C70R). Blood (2004 Mar 15) 103(6): 2407-9

10 US Census Bureau, International Data Base, 2004

11 Galan P, Yoon HC, Preziosi P, Viteri F, Valeix P, Fieux B, Briancon S, Malvy D, Roussel AM, Favier A, Hercberg S: Determining factors in the iron status of adult women in the SU.VI.MAX study. Supplementation en VItamines et Mineraux AntioXydants. Eur J Clin Nutr (1998 Jun) 52(6): 383-8

12 Diana J Fleming, Paul F Jacques, Katherine L Tucker, Joseph M Massaro, Ralph B D'Agostino, Sr, Peter WF Wilson and Richard J Wood: Iron status of the free-living, elderly Framingham Heart Study cohort: an ironreplete population with a high prevalence of elevated iron stores. American Journal of Clinical Nutrition, Vol. 73, No. 3, 638-646, March 2001

13 Logan S, Martins S, Gilbert R: Iron therapy for improving psychomotor development and cognitive function in children under the age of three with iron deficiency anaemia. Cochrane Database Syst Rev (2001)(2): CD001444
14 Grantham-McGregor S, Ani C: A review of studies on the effect of iron deficiency on cognitive development in children. J Nutr (2001 Feb) 131(2S-2): 649S-666S

15 Sabatine MS, Morrow DA, Giugliano RP, Burton PB J, Murphy SA, McCabe CH, Gibson $\mathrm{M}$, Braunwald $\mathrm{E}$ : Association of Hemoglobin Levels With Clinical Outcomes in Acute Coronary Syndromes. Circulation. 2005; 111: 0422049 doi: 10.1161/01.CIR.0000162477.70955.5F

16 Nikolsky E, Aymong ED, Halkin A, Grines CL, Cox DA, Garcia E, Mehran R, Tcheng JE, Grif fin JJ, Guagliumi G, Stuckey T, Turco M Cohen DA, Negoita M, Lansky AJ, Stone GW: Impact of anemia in patients with acute myocardial infarction undergoing primary percutaneous coronary intervention: analysis from the Controlled Abciximab and Device Investigation to Lower Late Angioplasty Complications (CADILLAC) Trial. J Am Coll Cardiol. 2004 Aug 4; 44(3): 547-53.

17 Cuervo LG, Mahomed K: Treatments for iron deficiency anaemia in pregnancy. Cochrane Database Syst Rev (2001)(2): CD003094

18 Makrides M, Crowther CA Gibson RA Gibson RS, Skeaff CM: Efficacy and tolerability of low-dose iron supplements during pregnancy: a randomized controlled trial. Am J Clin Nutr (2003 Jul) 78(1): 145-53

19 Nachtigall D: International Journal of Sports Medicine, "Iron deficiency in distance runners." October 1996; 17 (7): 473-9 quoted in Michelle Baldwin Iron Deficiency Anemia 2004. www.vanderbilt.edu/.../ IronDeficiencyAnemia.htm

20 Brownlie T, Utermohlen V, Hinton PS, Haas JD: Tissue iron deficiency without anemia impairs adaptation in endurance capacity after aerobic training in previously untrained women. Am J Clin Nutr (2004 Mar) 79(3): 437-43

21 Shaskey DJ, Green GA: Sports haematology Sports Med (2000 Jan) 29(1): 27-38

22 Beard J, Tobin B: Iron status and exercise. Am J Clin Nutr (2000 Aug) 72(2 Suppl): 594S-7S

23 Gasche C, Lomer MC, Cavill I, Weiss G: Iron anaemia, and inflammatory bowel diseases. Gut (2004 Aug) 53(8): 1190-7

24 Annibale B, Capurso G, Delle Fave G: The stomach and iron deficiency anaemia: a for gotten link. Dig Liver Dis (2003 Apr) 35(4): 288-95

25 Sargent JA, Acchiardo SR: Iron requirements in hemodialysis. Blood Purif (2004) 22(1): 112-23

26 Juarez-Vazquez J, Bonizzoni E, Scotti A: Iron plus folate is more effective than iron alone in the treatment of iron deficiency anaemia in pregnancy: a randomised, double blind clinical trial. BJOG (2002 Sep) 109(9): 1009-14

27 Verdon F, Burnand B. Stubi CL, Bonard C Graff M, Michaud A, Bischoff T, de Vevey $M$ Studer JP, Herzig L, Chapuis C, Tissot J Pecoud A, Favrat B:Iron supplementation for unexplained fatigue in non-anaemic women: double blind randomised placebo controlled trial. BMJ (2003 May 24) 326(7399): 1124

28 Duport N, Preziosi P, Boutron-Ruault MC Bertrais S, Galan P, Favier A, Lafond JL, Hercberg S: Consequences of iron depletion on health in menstruating women. Eur $\mathrm{J}$ Clin Nutr (2003 Sep) 57(9): 1169-75

29 Reffitt DM, Burden TJ, Seed PT, Wood J, Thompson RP, Powell JJ: Assessment of iron absorption from ferric trimaltol. Ann Clin Biochem (2000 Jul) 37 (Pt 4): 457-66

30 Whittaker $\mathrm{P}$ : Iron and zinc interactions in humans. Am J Clin Nutr (1998 Aug) 68(2 Suppl): 442S-446S
31 Schaefer JP, Tam Y, Hasinoff BB, Tawfik S, Peng $Y$, Reimche $L$, Campbell NR: Ferrous sulphate interacts with captopril. $\mathrm{Br} \mathrm{J}$ Clin Pharmacol (1998 Oct) 46(4): 377-81

32 Nelson M, Poulter J: Impact of tea drinking on iron status in the UK: a review. J Hum Nutr Diet (2004 Feb) 17(1): 43-54

33 Wilson JG, Lindquist JH, Grambow SC, Crook ED, Maher JF: Potential role of increased iron stores in diabetes. Am J Med Sci (2003 Jun) 325(6): 332-9

34 Rogers JT, Randall JD, Cahill CM, Eder PS, et al.: An Iron-responsive Element Type II in the 5'-Untranslated Region of the Alzheimer's Amyloid Precursor Protein Transcript J. Biol. Chem., November 22, 2002; Vol. 277, Issue 47, 45518-45528

35 Casadesus G, Smith MA, Zhu X, Aliev G, Cash AD, Honda K, Petersen RB, Perry G: Alzheimer disease: evidence for a central pathogenic role of iron-mediated reactive oxygen species. J Alzheimers Dis (2004 Apr) 6(2): 165-9

36 Honda K, Smith MA, Zhu X, Baus D, Merrick WC, Tartakoff AM, Hattier T, Harris PL, Siedlak SL, Fujioka H, Liu Q, Moreira PI, Miller FP, Nunomura A, Shimohama S, Perry G: Ribosomal RNA in Alzheimer disease is oxidized by bound redox-active iron. J Biol Chem. 2005 Jun 3; 280(22): 20978-86

37 Benvenisti-Zarom L, Chen J, Regan RF: The oxidative neurotoxicity of clioquinol. Neuropharmacology. 2005 Oct; 49(5): 687-94

38 Finefrock AE, Bush Al, Doraiswamy PM: Current status of metals as therapeutic targets in Alzheimer's disease. J Am Geriatr Soc (2003 Aug) 51(8): 1143-8

39 Gotz ME, Double K, Gerlach M, Youdim MB, Riederer $P$ : The relevance of iron in the pathogenesis of Parkinson's disease. Ann N Y Acad Sci (2004 Mar) 1012: 193-208

40 Moos T, Morgan EH: The metabolism of neuronal iron and its pathogenic role in neurological disease: review. Ann NY Acad Sci $(2004$ Mar) 1012: 14-26

41 Alpert PT: New and emerging theories of cardiovascular disease: infection and elevated iron. Biol Res Nurs (2004 Jul) 6(1): 3-10

42 Shah SV, Alam MG: Role of iron in atherosclerosis. Am J Kidney Dis (2003 Mar) 41(3 Suppl 1): S80-3

\section{Korrespondenzadresse:}

Prof. Dr. med. Reinhard Saller

UniversitätsSpital Zürich

Dep. für Innere Medizin

Institut für Naturheilkunde

Rämistrasse 100, CH-8091 Zürich

reinhard.saller@usz.ch 